\title{
CREATION, PHYSICAL AND CHEMICAL PROPERTIES OF ALKIL-2-((5-PHENETHYL-4-R-1,2,4- TRIAZOLE-3-YL)THIO)ACET(PROPAN,BENZ)IMIDATES
}

\author{
IGNATOVA TATYANA, KAPLAUSHENKO ANDRIY*, NAGORNA NATALIYA, AVRAMENKO ANTONINA
}

Department of Physical and Colloidal Chemistry, Farmaceutical Faculty, Zaporizhzhia State Medical University, Zaporizhzhia, Ukraine. Email: kaplaushenko@ukr.net

Received: 16 November 2018, Revised and Accepted: 10 January 2019

\section{ABSTRACT}

Objective: The aim of this research was to synthesize and evaluate physical-chemical properties of 5-pheneyhyl-4-R-3-thio-1,2,4-triazole and to establish identity and structure of the synthesized compounds, namely alkil-2-((5-phenethyl-4-R-1,2,4-triazole-3-yl)thio)acet(propan,benz)imidates.

Methods: As starting materials for synthesis of alkil-2-((5-phenethyl-4-R-1,2,4-triazole-3-yl)thio)acet(propan,benz)imidates, the corresponding 2-((5-phenethyl-4-R-1,2,4-triazole-3-yl)thio)aceto(propane,benzo)nitrile have been used. Synthesis has been set in the absolute alcohol medium (propanol or butanol alcohol) with chloroform, using the saturation with dry hydrogen chloride.

Results: In this study, we have developed nine new compounds have been received as a result of synthetic transformations, the structure of synthesized compounds has been confirmed by modern complex of physical-chemical methods of analysis ( ${ }^{1} \mathrm{H}$ NMR-spectroscopy, elemental analysis), and their individuality has been conducted on gas-liquid chromatograph Agilent 1260 Infinity HPLC equipped with a mass spectrometer Agilent 6120.

Conclusion: As a result of the work, it was synthesized the new compounds of the series of alkil-2-((5-phenethyl-4-R-1,2,4-triazole-3-yl)thio) acet(propan,benz)imidates and was characterized by elemental analysis and proton nuclear magnetic resonance spectroscopic analysis.

Keywords: 1,2,4-Triazole, Synthesis, Physical and chemical properties.

(C) 2019 The Authors. Published by Innovare Academic Sciences Pvt Ltd. This is an open access article under the CC BY license (http://creativecommons. org/licenses/by/4. 0/) DOI: http://dx.doi.org/10.22159/ajpcr.2019.v12i3.30763

\section{INTRODUCTION}

In the past three decades, a pharmaceutical market of Ukraine has had significant problems. This problem consists of a shortage of cheap national medicines, which has not its own side effects. The literature analysis of the last years about 1,2,4-triazole derivatives shows the intensive growth of bibliographic sources number [1-7]. Currently, the works of azole nitrogen compounds have been progressively getting people's attention and have been published, owing to their functionality and the wide range of the practical application [8-10].

More recently, the number of publications with the research of compounds, which contain 1,2,4-triazole core remains to grow $[1,11,12]$. 1,2,4-Triazoles are one very significant class of compounds for treating many types of diseases. For example, voriconazole, itraconazole, fluconazole, saperconazole, and other medicines with different biological activity [1-8]. Based on this, it was synthesized 1,2,4-triazole derivatives as new potential biological active substances.

According to the results of previous research, the purpose of our experiment is the creation and the establishment of physical-chemical properties of new highly efficient and low-toxic substances, namely alkil2-((5-phenethyl-4-R-1,2,4-triazole-3-yl)thio)acet(propan,benz)imidates.

\section{METHODS}

Physical and chemical properties of the new compounds (6-15) were considered according to the methods described in the State Pharmacopeia of Ukraine $[13,14]$. Melting point was measured by the capillary method (2.2.14). Elemental composition of compounds was received on the elemental analyzer Elementar Vario L cube (CHNS) (standard - Sulfonamide). Proton nuclear magnetic resonance $\left({ }^{1} \mathrm{H}\right.$ NMR) spectra of synthesized compounds are recorded in Varian Mercury VX-200 ( $\left.{ }^{1} \mathrm{H}, 200 \mathrm{MHz}\right)$. The solvent was used as DMSO-d6, the internal standart - as tetramethylsilane. Chromatography-mass spectrometry studies were conducted on gas-liquid chromatograph Agilent 1260 Infinity HPLC equipped with a mass spectrometer Agilent 6120 (in electrospray ionization).

General procedure for the preparation of compounds 6-15: Alkil2-((5-phenethyl-4-R-1,2,4-triazole-3-yl)thio)acet(propan,benz) imidates

The mixture of 2-((5-phenethyl-4-R-1,2,4-triazole-3-yl)thio) aceto(propane,benzo)nitrile $(0.01 \mathrm{~mol})$, chloroform $(10 \mathrm{ml})$ and absolute alcohol ( $25 \mathrm{ml})$ was added into Bunsen flask. It was equipped with calcium chloride tube. The mixture was cooled to the temperature of $-5^{\circ} \mathrm{C}$, and was saturated with dry hydrogen chloride. The 2 moles excess of dry hydrogen chloride was used for the saturation of 1 mole of 2-(c5-phenethyl-4-R1,2,4-triazole-3-yl)thio)aceto(propane,benzo)nitrile. Then, the mixture was left at $0^{\circ} \mathrm{C}$ for $24 \mathrm{~h}$ and the solvent was evaporated (Fig. 1).

Synthesized alkil-2-((5-phenethyl-4-R-1,2,4-triazole-3-yl)thio) acet(propan,benz)imidates (compounds 5-15, Tables 1-3) are brown $(6,7,8,11,15)$, white $(9,13)$, yellow $(10,14)$ or grey $(12)$ amorphous substances, soluble in organic solvents and slightly soluble in water. Target compounds were recrystallized from ethanol for the analysis.

\section{RESULTS AND DISCUSSION}

The individuality of alkil-2-((5-phenethyl-4-R-1,2,4-triazole-3yl)thio)acet(propan,benz)imidates was confirmed by gas-liquid chromatography Agilent 1260 Infinity HPLC equipped with a mass spectrometer Agilent 6120 . The melting point was determined by the capillary method (2.2.14) with the PTP (M) device (Table 1).

The structure of synthesized compounds (6-15) was confirmed by the complex using of elemental analysis. The elemental composition of new substances coincides within the limits of the calculated data error (Table 2). 


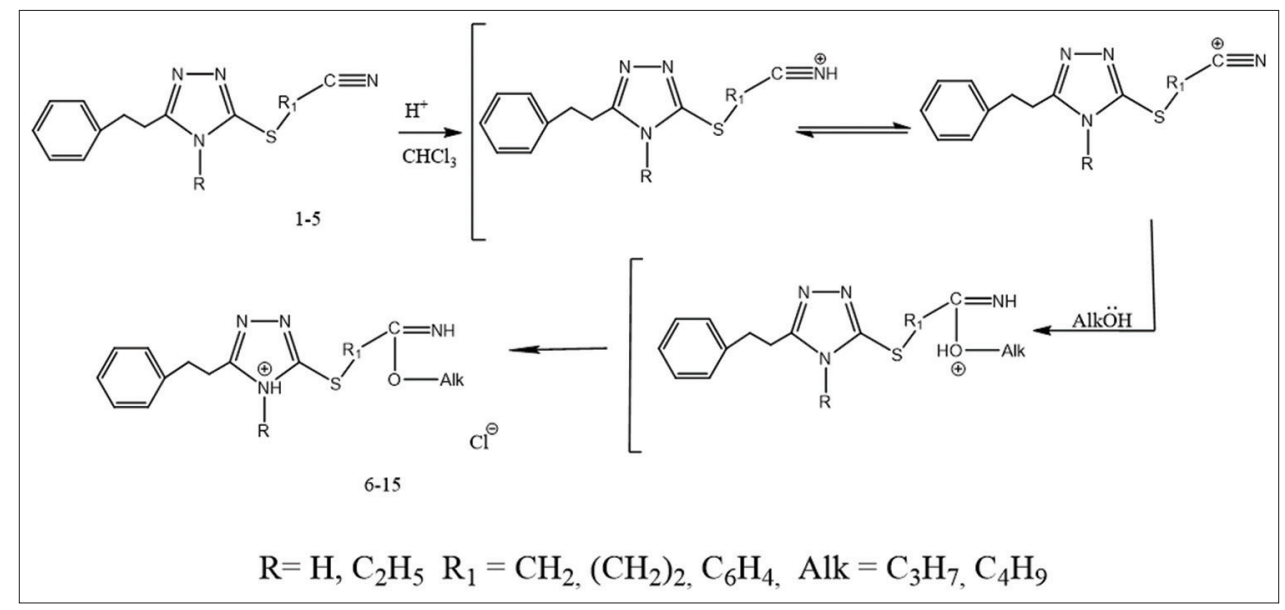

Fig. 1: Scheme of synthesis of alkil-2-((5-phenethyl-4-R-1,2,4-triazole-3-yl)thio)acet(propan,benz)imidates

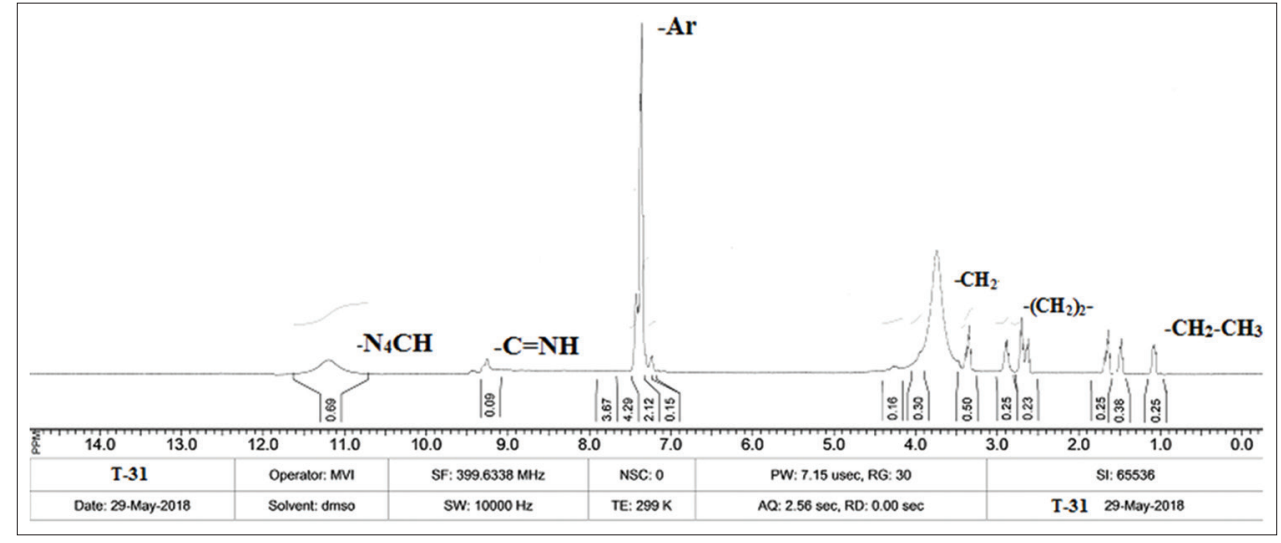

Fig. 2: ${ }^{1} \mathrm{H}$ NMR spectrum of propyl 2-((5-phenethyl-4H-1,2,4-triazole-3-yl)thio)acetimidate

Table 1: Physical-chemical properties of compounds 6-15

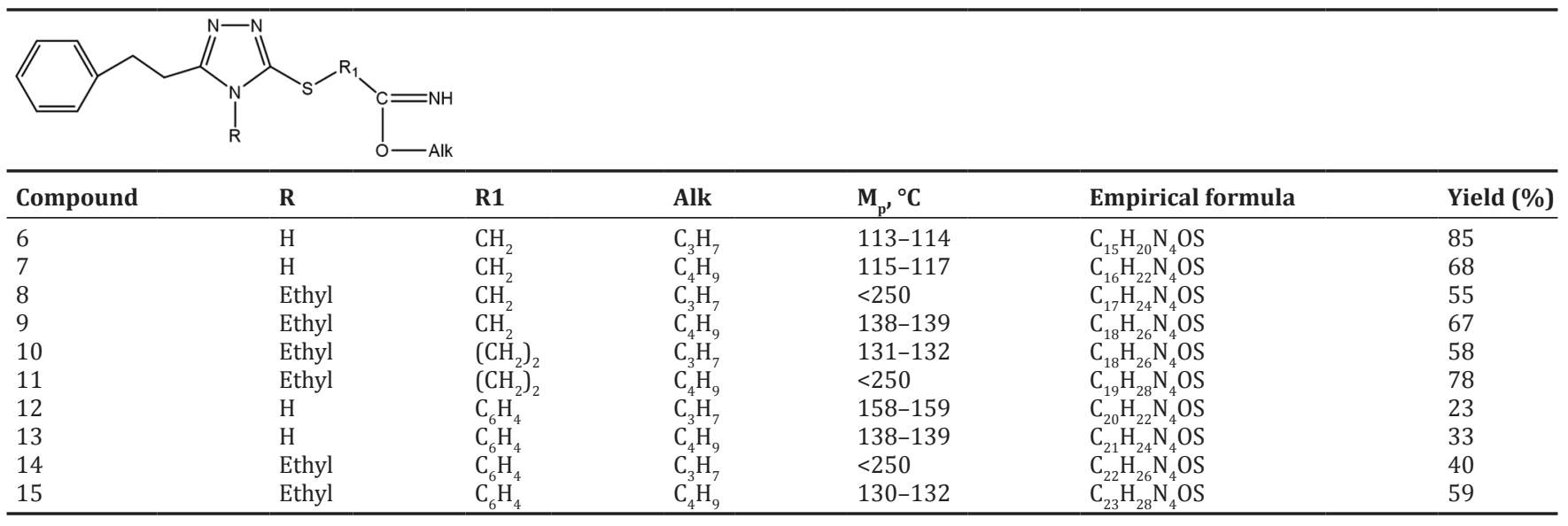

The results of proton nuclear magnetic resonance spectra of synthesized compounds are presented in Table 3. ${ }^{1} \mathrm{H}$ NMR spectrum of propyl 2-((5-phenethyl-4H-1,2,4-triazole-3-yl)thio)acetimidate are presented in Fig. 2. All experimental values of ${ }^{1} \mathrm{H}$ NMR-spectrophotometry of new alkil-2-((5-phenethyl-4-R-1,2,4-triazole-3-yl)thio)acet(propan,benz) imidates correspond to theoretical data $[15,16]$.

\section{CONCLUSIONS}

An effective method of new 1,2,4-triazole derivatives was developed. A series of alkil-2-((5-phenethyl-4-R-1,2,4-triazole-3- yl)thio)acet(propan,benz)imidates was successfully synthesized and characterized by elemental analysis and proton nuclear magnetic resonance spectroscopic analysis. These compounds can be used for modeling chemical molecules of new biologically active substances.

\section{ACKNOWLEDGMENTS}

We would like to acknowledge complete support from the Zaporizhizhia State Medical University. We thank rector of Zaporizhizhia State Medical University Prof. Yu. M. Kolesnyk for his help and support. 
Table 2: The results of elemental composition determination of compounds 6-15

\begin{tabular}{|c|c|c|c|c|c|c|c|c|}
\hline \multirow[t]{2}{*}{ Compound } & \multicolumn{4}{|c|}{ Found (\%) } & \multicolumn{4}{|c|}{ Calculated (\%) } \\
\hline & C & $\mathbf{H}$ & $\mathbf{N}$ & S & C & H & $\mathbf{N}$ & S \\
\hline 6 & 51.19 & 6.64 & 18.42 & 10.54 & 51.18 & 6.62 & 18.41 & 10.53 \\
\hline 7 & 60.37 & 6.97 & 17.57 & 10.09 & 60.35 & 6.96 & 17.59 & 10.07 \\
\hline 8 & 61.44 & 7.29 & 16.85 & 9.62 & 61.42 & 7.28 & 16.85 & 9.64 \\
\hline 9 & 62.42 & 7.54 & 16.19 & 9.23 & 62.40 & 7.56 & 16.17 & 9.25 \\
\hline 10 & 62.42 & 7.58 & 16.15 & 9.26 & 62.40 & 7.56 & 16.17 & 9.25 \\
\hline 11 & 63.32 & 7.84 & 15.52 & 8.90 & 63.30 & 7.83 & 15.54 & 8.89 \\
\hline 12 & 65.57 & 6.07 & 15.27 & 8.76 & 65.55 & 6.05 & 15.29 & 8.75 \\
\hline 13 & 66.31 & 6.37 & 14.73 & 8.44 & 66.29 & 6.36 & 14.72 & 8.43 \\
\hline 14 & 66.96 & 6.62 & 14.22 & 8.11 & 66.98 & 6.64 & 14.20 & 8.13 \\
\hline 15 & 67.63 & 6.92 & 13.72 & 7.86 & 67.62 & 6.91 & 13.71 & 7.85 \\
\hline
\end{tabular}

Table 3: ${ }^{1} \mathrm{H}$ NMR spectra of compounds 6-15

\begin{tabular}{|c|c|}
\hline Compound & ${ }^{1} \mathrm{H}$ NMR $(\delta, \mathrm{ppm}, \mathrm{TMS})$ \\
\hline 6 & $\begin{array}{l}\left.\text { 1.05-1.70 (5H, m, } \mathrm{CH}_{2}-\mathrm{CH}_{3}\right) ; 2.80-2.95\left(4 \mathrm{H}, \mathrm{m},\left(\mathrm{CH}_{2}\right)_{2}\right) ; 3.60-3.85\left(4 \mathrm{H}, \mathrm{s}, \mathrm{CH}_{2}\right) ; 7.25-7.30\left(5 \mathrm{H}, \mathrm{m}, \mathrm{C}_{6} \mathrm{H}_{5}\right) ; 9.45(1 \mathrm{H}, \mathrm{s}, \mathrm{NH}=\mathrm{C}) ; \\
11.20\left(1 \mathrm{H}, \mathrm{s}, \mathrm{N}_{4} \mathrm{CH}\right)\end{array}$ \\
\hline 7 & $\begin{array}{l}0.85-1.55\left(7 \mathrm{H}, \mathrm{m}, \mathrm{CH}_{2}-\mathrm{CH}_{2}-\mathrm{CH}_{3}\right) ; 2.85-2.90\left(4 \mathrm{H}, \mathrm{m},\left(\mathrm{CH}_{2}\right)_{2}\right) ; 3.60-3.80\left(4 \mathrm{H}, \mathrm{s}, \mathrm{CH}_{2}\right) ; 7.27-7.33\left(5 \mathrm{H}, \mathrm{m}, \mathrm{C}_{6} \mathrm{H}_{5}\right) ; 9.40(1 \mathrm{H}, \mathrm{s}, \mathrm{NH}=\mathrm{C}) \\
11.15\left(1 \mathrm{H}, \mathrm{s}, \mathrm{N}_{4} \mathrm{CH}\right)\end{array}$ \\
\hline 8 & $1.10-1.40\left(6 \mathrm{H}, \mathrm{m}, \mathrm{CH}_{3}\right) ; 1.80\left(2 \mathrm{H}, \mathrm{m}, \mathrm{CH}_{2}\right) ; 2.85-2.90\left(4 \mathrm{H}, \mathrm{m},\left(\mathrm{CH}_{2}\right)_{2}\right) ; 3.64-4.15\left(6 \mathrm{H}, \mathrm{m}, \mathrm{CH}_{2}\right) ; 7.15-7.20\left(5 \mathrm{H}, \mathrm{m}, \mathrm{C}_{6} \mathrm{H}_{5}\right) ; 9.30(1 \mathrm{H}, \mathrm{s}, \mathrm{NH}=\mathrm{C})$ \\
\hline 9 & $1.07-1.35\left(6 \mathrm{H}, \mathrm{m}, \mathrm{CH}_{3}\right) ; 1.45-2.90\left(8 \mathrm{H}, \mathrm{m},\left(\mathrm{CH}_{2}\right)_{2}\right) ; 3.45-4.18\left(6 \mathrm{H}, \mathrm{m}, \mathrm{CH}_{2}\right) ; 7.23-7.42\left(5 \mathrm{H}, \mathrm{m}, \mathrm{C}_{6} \mathrm{H}_{5}\right) ; 9.46(1 \mathrm{H}, \mathrm{s}, \mathrm{NH}=\mathrm{C})$ \\
\hline 10 & $0.75-1.25\left(6 \mathrm{H}, \mathrm{m}, \mathrm{CH}_{3}\right) ; 1.75-4.10\left(14 \mathrm{H}, \mathrm{m}, \mathrm{CH}_{2}\right) ; 7.10-7.24\left(5 \mathrm{H}, \mathrm{m}, \mathrm{C}_{6} \mathrm{H}_{5}\right) ; 9.40(1 \mathrm{H}, \mathrm{s}, \mathrm{NH}=\mathrm{C})$ \\
\hline 11 & $0.75-1.45\left(6 \mathrm{H}, \mathrm{m}, \mathrm{CH}_{3}\right) ; 1.50-4.15\left(16 \mathrm{H}, \mathrm{m}, \mathrm{CH}_{2}\right) ; 7.21-7.50\left(5 \mathrm{H}, \mathrm{m}, \mathrm{C}_{6}^{0} \mathrm{H}_{5}\right) ; 9.43(1 \mathrm{H}, \mathrm{s}, \mathrm{NH}=\mathrm{C})$ \\
\hline 12 & $1.35\left(3 \mathrm{H}, \mathrm{m}, \mathrm{CH}_{3}\right) ; 1.70-3.60\left(8 \mathrm{H}, \mathrm{m}, \mathrm{CH}_{2}\right) ; 7.15-7.35\left(4 \mathrm{H}, \mathrm{m}, \mathrm{C}_{6} \mathrm{H}_{4}\right) ; 7.40-7.50\left(5 \mathrm{H}, \mathrm{m}, \mathrm{C}_{6} \mathrm{H}_{5}\right) ; 9.50(1 \mathrm{H}, \mathrm{s}, \mathrm{NH}=\mathrm{C}) ; 11.20\left(1 \mathrm{H}, \mathrm{s}, \mathrm{N}_{4} \mathrm{CH}\right)$ \\
\hline 13 & $0.95\left(3 \mathrm{H}, \mathrm{m}, \mathrm{CH}_{3}^{3}\right) ; 1.45-3.55\left(10 \mathrm{H}, \mathrm{m}, \mathrm{CH}_{2}\right) ; 7.10-7.40\left(4 \mathrm{H}, \mathrm{m}, \mathrm{C}_{6} \mathrm{H}_{4}\right) ; 7.15-7.20\left(5 \mathrm{H}, \mathrm{m}, \mathrm{C}_{6} \mathrm{H}_{5}\right) ; 9.45(1 \mathrm{H}, \mathrm{s}, \mathrm{NH}=\mathrm{C}) ; 11.20\left(1 \mathrm{H}, \mathrm{s}, \mathrm{N}_{4} \mathrm{CH}\right)$ \\
\hline 14 & $1.10-1.35\left(6 \mathrm{H}, \mathrm{m}, \mathrm{CH}_{3}\right) ; 1.50-4.24\left(10 \mathrm{H}, \mathrm{m}, \mathrm{CH}_{2}\right) ; 7.15-7.30\left(4 \mathrm{H}, \mathrm{m}, \mathrm{C}_{6} \mathrm{H}_{4}\right) ; 7.20-7.35\left(5 \mathrm{H}, \mathrm{m}_{6} \mathrm{C}_{6} \mathrm{H}_{5}\right) ; 9.55(1 \mathrm{H}, \mathrm{s}, \mathrm{NH}=\mathrm{C})$ \\
\hline 15 & $1.05-1.35\left(6 \mathrm{H}, \mathrm{m}, \mathrm{CH}_{3}^{3}\right) ; 1.45-4.20\left(10 \mathrm{H}, \mathrm{m}, \mathrm{CH}_{2}\right) ; 7.20-7.45\left(4 \mathrm{H}, \mathrm{m}, \mathrm{C}_{6}^{\circ} \mathrm{H}_{4}\right) ; 7.25-7.30\left(5 \mathrm{H}, \mathrm{m}, \mathrm{C}_{6}^{6} \mathrm{H}_{5}\right) ; 9.45(1 \mathrm{H}, \mathrm{s}, \mathrm{NH}=\mathrm{C})$ \\
\hline
\end{tabular}

\section{AUTHORS' CONTRIBUTIONS}

The article is a product of the intellectual environment of the whole team and that all members have contributed in various degrees to the synthesis, physical-chemical methods used, to the research concept, and to the experiment design.

\section{CONFLICTS OF INTEREST}

All authors have none to declare.

\section{REFERENCES}

1. Kaplaushenko AG, Samelyuk YG, Shcherbak MO, Benjamini Y, Hochberg Y. Practical Value and Application of 1, 2, 4-Triazole Derivatives. Zaporizhzhia: Karat; 2016.

2. Samelyuk YG. Synthesis and Study of Biologically Active Derivatives of 1, 2, 4-triazole-3-Thione Containing Methoxyphenyl Substituents. PhD Dissertation. Zaporizhzhia; 2016. Available from: http://www. dspace.zsmu.edu.ua/handle/123456789/5238.

3. Shcherbak MO. Research Synthetic, Physical, Chemical and Biological Properties of 4-Amino-5-(2, 3, 4-Nitrophenyl)-1, 2, 4-Triazole-3-Thiones and their $\mathrm{N}$ and S-Substituted. PhD Dissertation. Zaporizhzhia; 2015. Available from: http://www.dspace.zsmu.edu.ua/ handle/123456789/3570.

4. Madhavi K, Ramya GS. Synthesis, antioxidant and anti-inflammatory activities of ethyl 2-(2-cyano-3-(substituted phenyl) acrylamido)-4, 5-dimethylthiophene-3-carboxylates. Asian J Pharm Clin Res 2017;10:95-100.

5. Kaplaushenko TM, Panasenko OI, Kucheryavy YM. Research of the synthetic, physical and chemical properties of 3-alkylsulfonyl-5(chinoline-2-yl, 2-hydroxychinoline-4-yl)-4-R1-2,4-dihydro-3N-1,2,4triazoles. Asian J Pharm Clin Res 2017;10:81-3.
6. Monther FM, Noor HN, Nethal HH. Synthesis and preliminary pharmacological evaluation of new naproxen analogues having 1,2, 4-triazole-3-thiol. Int J Pharm Pharm Sci 2017;9:66-71.

7. Santoshkumar S, Satyanarayan ND, Anantacharya R, Sameer P. Synthesis, antimicrobial, antitubercular and cheminformatic studies of 2-(1-benzofuran-2-yl)-N'-[(3Z)-2-oxo-1,2-dihydro-3H-indol-3-ylidene] quinoline-4-carbohydrazide and its derivatives. Int J Pharm Pharm Sci 2017;9:260-7.

8. Shcherbina RO. Synthesis, Transformation, Physical, Chemical and Biological Properties of Derivatives Of 2-(4H-1, 2, 4-triazol-3-ylthio) acetaldehyde: Dr. Farm. Sci. Diss. Zaporizhzhia: 2014.

9. Gulina YS, Kaplaushenko AG. Synthesis and physical-chemical properties of 2-(5-(1H-tetrazole-1-ylmethyl)-4-R-4H-1,2,4-triazole-3ylthio)acetate(propane), 2, 4 (5-(1H-tetrazole-1-ylmethyl)-4-phenyl$4 H-1,2,4$-triazole-3-ylthiomethyl)-benzoic acids and their salts. Curr Issues Pharm Med 2016;2:32-7.

10. Kaplaushenko TM, Panasenko OI. Synthesis and physical-chemical properties of 3-alkylthio-5-(quinoline-2-yl, 2-hydroxyquinoline-4yl)-4-R-2, 4-dihydro-3H-1, 2, 4-triazoles. Zaporizhia Med J 2016;3: 99-103.

11. Hulina YS, Kaplaushenko AG. Synthesis, physical and chemical properties of 5-((1H-tetrazole-1-yl) methyl)-4-R-4H-1, 2, 4-triazole-3thiols and their chemical transformations. Biopharm J 2018;1:26-30.

12. Kaplaushenko TM. Synthesis, physical-chemical properties and further transformation of 5-(quinoline-2-yl, 2-hydroxyquinoline-4-yl)-4-R-2, 4-dyhydro-3H-1, 2, 4-triazole-3-thiones. Curr Issues Pharm Med 2016;2:20-5

13. The State Pharmacopoeia of Ukraine. Kharkov: RIREG; 2001.

14. The State Pharmacopoeia of Ukraine. Kharkov: RIREG; 2004.

15. Kazicyna LA. Application of UV, IR, NMR and Mass Spectroscopy in Organic Chemistry. Moscow: Mosk un-ty; 1979.

16. Nifantev IE, Ivchenko PV. Practical Course of Nuclear Magnetic Resonance Spectroscopy. Moscow: Mosk un-ty; 2006. 